\title{
MATERIAL DOCENTE PARA LA VISIBILIZACIÓN DE MODELOS DE REFERENCIA EN EL EMPRENDIMIENTO FEMENINO. CONCIENCIANDO EN EL AUTOEMPLEO
}

\section{TEACHING MATERIAL FOR THE VISIBILIZATION OF MODELS IN THE FEMALE ENTREPRENEURSHIP. RAISING AWARENESS IN SELF-EMPLOYMENT}

\author{
Pablo Rodríguez Gutiérrez ${ }^{1}$ \\ pablo.rodriguez@uco.es \\ Sandra $\mathrm{M}^{\mathrm{a}}$ Sánchez Cañizares ${ }^{1}$ \\ Fernando J. Fuentes García ${ }^{1}$ \\ Mercedes Luque Vílchez ${ }^{2}$ \\ Paula Rodríguez Torrico ${ }^{2}$ \\ Guzmán A. Muñoz Fernández ${ }^{1}$ \\ Luna Santos Roldán ${ }^{1}$ \\ Julia M. Núñez Tabales ${ }^{1}$ \\ Francisco Rey Carmona ${ }^{1}$ \\ Universidad de Córdoba ${ }^{1}$ \\ Universidad de Burgos ${ }^{2}$
}

\begin{abstract}
In a time of great changes, university teaching is not alien to the challenges and needs that the society in which it develops its activity poses. The role of women as a dynamic agent of economic activity plays a special role. In this sense, the university should try to foster an entrepreneurial spirit among the students. According to this, this project is oriented towards the development of teaching material for the visibility of female entrepreneurship cases. The teaching material has materialized in a book in editing process coedited by the social council of the University of Córdoba and the publishing house Tirant lo Blanc (indexed in the first quartile of the SPI index). This material provides a selection of real and varied cases that the student can consult, thus becoming an essential didactic complement in the Business Creation Course. In the medium term, this book, through its continuous use in future courses, could allow to track and measure response rates entrepreneurial intention among students.
\end{abstract}

Keywords: Entrepreneurship culture, Teaching innovation, Woman.

\section{Resumen}

En un momento de grandes cambios, la docencia universitaria no se encuentra ajena a los retos y necesidades planteados por la sociedad en la que desenvuelve su actividad. Juega una especial importancia el papel de la mujer como agente dinamizador de la actividad económica. En este sentido la universidad debe de tratar de fomentar el espíritu emprendedor entre el alumnado. Según esto, este proyecto se orienta hacia la elaboración de material docente para la mejora de la visibilización del rol empresarial de la mujer mediante la elaboración de casos de emprendimiento femenino. El material docente se ha materializado en un libro coeditado por el Consejo Social de la universidad de Córdoba, la Fundación Caja Rural del Sur y la editorial Tirant lo Blanc (indexada en el primer cuartil del índice SPI). Dicho material proporciona una variada selección de casos reales que el alumno puede consultar convirtiéndose así en un complemento didáctico esencial en la Asignatura de Creación de Empresas. Además, a medio plazo, este libro, mediante su continua utilización en próximos cursos, podría permitir hacer un seguimiento y medición de las tasas de respuesta en intención emprendedora entre los alumnos.

Palabras clave: Cultura emprendedora, Innovación docente, emprendimiento, mujer.

\section{INTRODUCCIÓN}

Las mujeres constituyen un sujeto especialmente relevante para el desarrollo económico y social de nuestro país en los últimos años. Sin embargo, en relación a la actividad emprendedora, su presencia es aún reducida en relación a la de los hombres. A pesar de que el crecimiento de la actividad empresarial femenina y la implicación de las mujeres en actividades económicas son notables, aún es elevada la brecha que separa el grado de emprendimiento entre hombres y mujeres. El emprendimiento ha adquirido un papel cada vez más protagonista, 
como motor de modernización y sostenibilidad gracias a la creación de nuevas empresas que, en muchas ocasiones, implementan nuevos modelos de negocio distintos de los tradicionales.

Desde la esfera política el impulso de la actividad emprendedora ha sido una constante en la política de empleo de la Unión Europea (UE) al ser considerada una palanca de innovación, competitividad y crecimiento. Por otra parte las actividades de este proyecto se enmarcan dentro del Objetivo número 5 de los Objetivos de Desarrollo Sostenible de Naciones Unidas (Naciones Unidas, 2015), mediante el que se busca "lograr la igualdad entre los géneros y empoderar a todas las mujeres y las niñas" y más concretamente como uno de los objetivos principales "Velar por la participación plena y efectiva de las mujeres y la igualdad de oportunidades de liderazgo a todos los niveles de la adopción de decisiones en la vida política, económica y pública".

Respecto al género, la literatura que estudia la relación entre este y la creación de nuevas empresas ponen de manifiesto una constante relación entre el hecho de ser hombre y el deseo o la intención de crear una empresa (Díaz et al., 2007; Veciana et al., 2005). El Informe Ejecutivo GEM España 2014 se hace eco del menor nivel de incorporaciones de la mujer a la actividad emprendedora. En este sentido, los datos muestran que un $60 \%$ de las iniciativas empresariales son adoptadas por hombres.

En lo que se refiere a la actitud hacia el emprendimiento entre los universitarios, encontramos evidencias sobre el perfil del emprendedor, y en concreto en estudiantes universitarios, donde queda de manifiesto la menor propensión de la mujer a emprender (Cañizares y Fuentes, 2010: Hermnangomez et al. 2006; Lim y Envick 2013; Ventura y Quero 2013; Wilson et al. 2007). Entre los factores explicativos, siguiendo el enfoque del estudio del entorno como factor explicativo del diferencial de emprendimiento entre hombres y mujeres, Baughn et al. (2006) y Lagowitz y Minniti (2007) afirman que en sociedades dónde el rol de la mujer está muy ligado a las responsabilidades familiares, la actividad emprendedora se percibe como menos deseable entre ellas.

En relación a este último hecho, consideramos que la falta de modelos de referencia de emprendedoras puede dificultar la labor de creación de empresas por parte de las mujeres (Justo y Diaz, 2012), puesto que ellas, con mayor frecuencia que los hombres, tendrán un modelo de referencia incompleto, bien porque el género es distinto o bien porque los rasgos del modelo serán insuficientes para poder servirles de guía. Sin embargo, la construcción de un modelo de referencia no se hace sobre una persona real, sino sobre percepciones personales de personas reales, sesgadas por la importancia diferencial que se le dé a determinadas características de las mismas.

Relacionado con esto, Minniti (2004) ha analizado el aumento de la confianza de los individuos generada por la existencia de precedentes y modelos a seguir, y el efecto que su conocimiento tiene en la reducción de la ambigüedad en el entorno del potencial emprendedor. Así mismo, se confirma la influencia que puede tener la falta de modelos de referencia emprendedora en el bajo porcentaje de emprendimiento de diferentes colectivos, como el femenino.

De esta forma, estaría relacionado con los prejuicios de género, por lo que apoyan la tesis de que son necesarias materias específicas de género en las enseñanzas universitarias que ayuden a derribar dichos prejuicios y estereotipos.

A pesar de que el crecimiento de la actividad empresarial femenina y la implicación de las mujeres en actividades económicas son notables, aún existe una elevada brecha que separa el grado de emprendimiento entre hombres y mujeres.

En respuesta a esta necesidad, se ha llevado a cabo un proyecto de elaboración de material docente para la visibilización de casos de emprendimiento femenino, que se enmarca dentro de la línea de innovación docente "3" que busca impulsar la igualdad de género en la docencia universitaria. Ésta se alínea con una de las principales competencias a desarrollar la "Competencia universidad 3 (CU3): Potenciar los hábitos de búsqueda activa de empleo y la capacidad de emprendimiento". Por otra parte se puso en práctica uno de los principales ejes temáticos formulados por el I Plan de Igualdad entre Hombres y Mujeres de la UCO, uno de los cuales está dedicado a fomentar la perspectiva de género en la docencia.

\section{EMPRENDIMIENTO FEMENINO DE CARÁCTER SOCIAL}

En los últimos años estamos asistiendo a cambios profundos en el tejido económico y social de nuestro país. Tras una intensa destrucción de empleo, sobre todo desde el año 2008, se ha vuelto a la senda del crecimiento económico, pero a costa del incremento del empleo temporal y la reducción de salarios. En determinadas situaciones, el salario es tan precario que no cubre los gastos aceptables de una vida digna. En comparación con Europa, nuestro país registra una de las cifras más altas de temporalidad juvenil, situándose en 2011 en un 61,4\% de los empleos. En junio de 2017, según los datos del Servicio Público de Empleo Estatal, el porcentaje de contratos temporales sobre el total es de un $92 \%$. 
Este proceso económico ha tenido a la vez su traducción en políticas de austeridad donde los gobiernos encuentran dificultades para mantener y garantizar a la ciudadanía el estado de bienestar. Esto provoca una reducción del gasto público plasmado en escasez de servicios públicos, reducción de prestaciones sociales, eliminación de determinados derechos de la ciudadanía, incremento de impuestos, en especial, a rentas bajas, y una reducción en áreas sociales como son la sanidad y educación, entre otras. Todo ello ha generado un clima de gran desconfianza social y un cuestionamiento del conjunto del sistema.

La identificación de estos datos hace que la Comisión determine la necesidad de desarrollar políticas que consigan en última instancia una mayor inclusión social. Siguiendo el marco de esta Plataforma Europea contra la pobreza y exclusión social, la Comisión tiende a favorecer un modelo de emprendimiento que persiga la responsabilidad social de las empresas. De esta forma se reconoce un concepto de "empresa" en el que se distinguen multitud de modelos. Concretamente, se reconoce la necesidad de "crear un ecosistema para promover las empresas e iniciativas sociales" donde lo importante es la capacidad de innovación de los emprendedores.

A pesar de que España cuenta con una amplia tradición en lo referente a economía social, paradójicamente, cuenta con un bajo número de emprendimiento en este tipo de negocios, llegando a ser uno de los niveles más bajos de Europa.

\section{OBJETIVOS}

Debido a que en la asignatura de Creación de Empresas una parte importante del material está enfocado al aprendizaje y desarrollo de capacidades y habilidades (liderazgo, trabajo en equipo, gestión de emociones, etc.), la aplicación de un enfoque de género resulta fundamental. En concreto, el desarollo de esta actividad se realizó para el ámbito del estudio de las asignaturas siguientes:

- Creación de Empresas, dentro del plan docente de los grados en Administración y Dirección de Empresas y Doble Grado en Derecho y Administración de Empresas.

- Creación de Empresas Turísticas, Grado en Turismo.

- Creación de Empresas Culturales, Grado de Gestión Cultural.

El objetivo fundamental del proyecto se centra en la puesta a disposición de los alumnos de la asignatura de un libro en formato electrónico (o impreso) con diferentes casos de estudio donde se presentan diferentes problemáticas relacionadas con el proceso de creación de una empresa y que afectan de forma particular a mujeres emprendedoras. De este modo se pretende potenciar la calidad de la docencia de la asignatura, mejorando la sensibilización del alumnado en cuestiones de género, visibilizando el papel de la mujer en el ámbito empresarial, así como desarrollando competencias transversales como son el empredimiento y la autonomía personal en la toma de decisiones. Los objetivos concretos del proyecto fueron los siguientes:

- Elaboración de un libro de casos prácticos donde se estudian diferentes sitiaciones reales, tratadas de manera teórica en las sesiones presenciales de la asignatural.

- Fomento de la utilización de los diferentes casos de estudio desarrollados en el libro y de los recursos didácticos asociados a ellos (videos, enlaces web).

\section{Metodología}

Los objetivos previamente expuestos fueron desarrollados por medio del método del caso (Yin, 2003). Se analizaron varios casos de emprendimiento fememino que configuran una selección que el alumno puede consultar. De acuerdo con Van Dam (2004) la retención del conocimiento entre el alumnado es significativamente superior mediante del empleo de métodos basados en la experiencia, y no tanto aquellos métodos de formación basados en la clase magistral o, incluso, el trabajo de casos de estudio previamente elaborados.

La información necesaria para el análisis de los casos fue recabada por medio de entrevistas semiestructuradas (Berg y Lune, 2012). En las entrevistas las mujeres emprendedoras de diversos ámbitos

1 COM (2011) Bruselas 25.10.2011 - COMUNICACIÓN DE LA COMISIÓN AL PARLAMENTO EUROPEO, AL CONSEJO, AL COMITÉ ECONÓMICO Y SOCIAL EUROPEO Y AL COMITÉ DE LAS REGIONES. Iniciativa en favor del emprendimiento social. 
narraron su experiencia y transmitieron los problemas a los que se han enfrentado, cómo han luchado por su idea de negocio y las motivaciones que les llevaron a optar por esa forma de vida.

\section{RESULTADOS OBTENIDOS Y DISCUSIÓN}

\section{$1^{a}$ - Identificación de casos y puesta en contacto con las emprendedoras}

Los casos comprendidos en el trabajo son los siguientes:

\begin{tabular}{lll}
\hline Caso / empresa & Sector & Localización \\
\hline Cynara Asesores & Asesoramiento profesional & Córdoba \\
Hoy no cocino & Hostelería / Restauración & Madrid \\
Gliquet & Nuevas tecnologías & Valencia \\
Sopinet & Nuevas tecnologías & Córdoba \\
Asofi Asesores & Servicios profesionales & León \\
Las Aventuras de Tate & Nuevas tecnologías & Córdoba \\
Gourmet Jienense & Hostelería / Restauración & Jaén \\
Innovación Creativa & Nuevas tecnologías & Córdoba \\
La voz de Marta & Servicios profesionales & Córdoba \\
*Pollos de la aldea & Sector primario / emprendimiento social & Valladolid \\
$*$ La otra cafetería & La otra cafetería / emprendimiento social & Valladolid \\
\hline
\end{tabular}
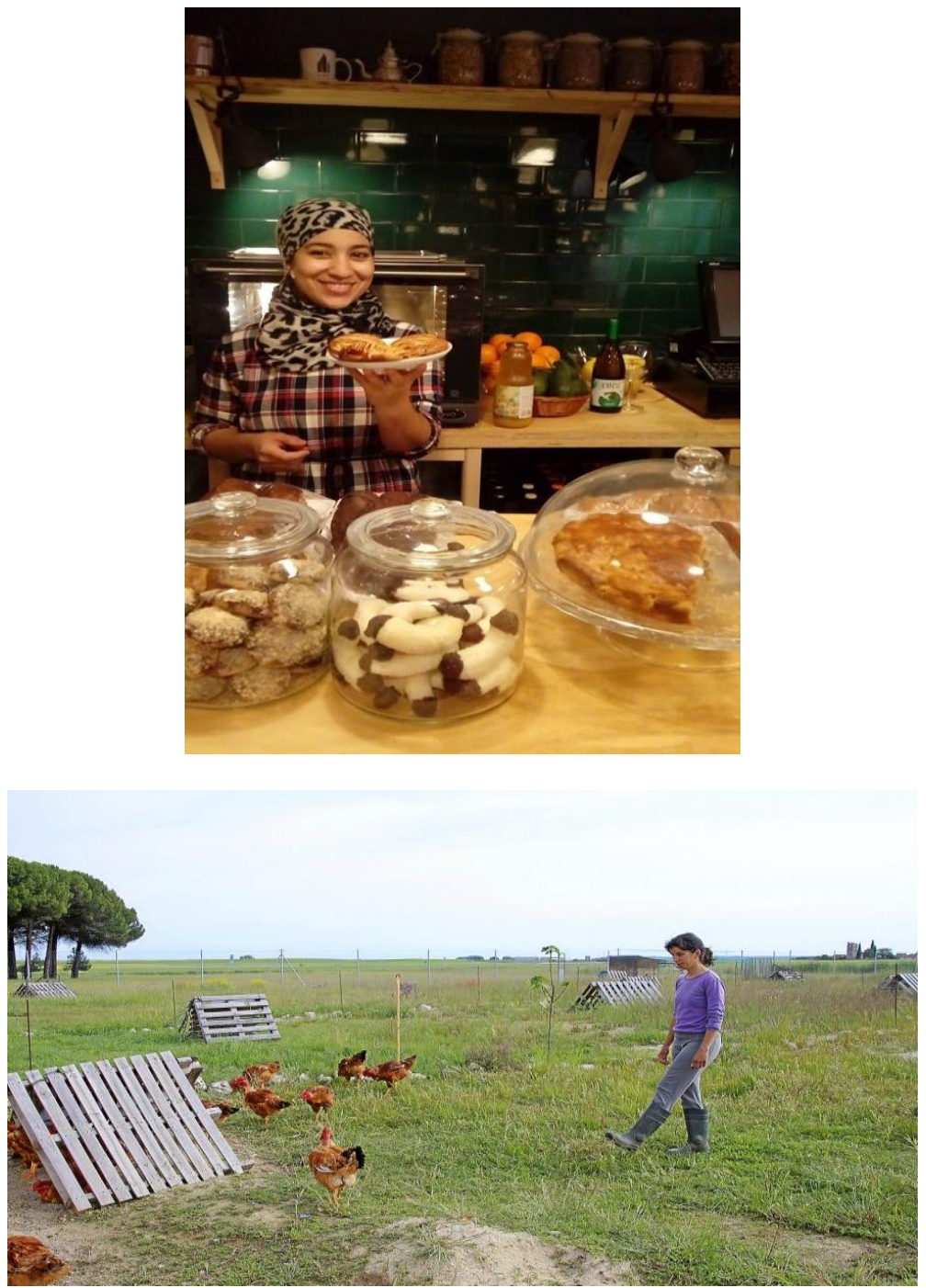

1. Imágenes de algunas de las emprendedoras en sus negocios. 
Los dos casos de emprendimiento social femenino que se proponen (Pollos de la aldea y La otra cafetería), aúnan dos rasgos identificados: por un lado, el hecho de que sus objetivos cuenten con una naturaleza de corte social; a la vez que la mujer es la protagonista en estos proyectos. Una de ellas lo inicia como proyecto individual, aunque con numerosas implicaciones del territorio donde se asienta, y el otro emprendimiento refleja los valores del cooperativismo y la ayuda mutua dentro de un colectivo social específico como es el de las mujeres que pretenden romper con las barreras y estereotipos de género.

Uno de los emprendimientos, "Pollos de la Aldea" nace desde la situación de desempleo de la empresaria. Importante la implicación de numerosos vecinos de la localidad produciendo cambios sociales, creatividad en las infraestructuras e instalación de toda su familia donde está localizado su negocio. Este negocio consigue que la empresaria realice un trabajo emocionalmente importante para ella. Además, de un desarrollo rural logra una implicación colectiva, uniendo su negocio a otros elementos de la economía solidaria (bioconstrucción). Todo esto dentro de las dificultades propias de emprender en el mundo rural, con el condicionante de género que agrava más el problema.

El otro emprendimiento que se detalla en esta sección "La otra librería. Cafetería" es una idea colectiva, y se puede enmarcar como "proyecto de vida". Es una idea innovadora, con una idea sencilla y sostenible dentro de una ciudad. Representa un modelo económico inclusivo e integrador.

Ambos casos comparten el problema añadido de la obtención de fuentes de financiación externas. En los dos casos de emprendimiento social se da la característica de búsqueda de apoyo económico dentro de un modelo de cooperación en red. Captan recursos financieros a través de un modelo de "financiación social". Esta red se denomina "Asociación Fiare Castilla y León (Asociación Fomento de alternativas para la articulación de la Responsabilidad Económica en Castilla y León)", que se constituye a través de personas dispuestas a apoyar la economía solidaria. La Asociación Fiare acerca a demandantes y ahorradores responsables, que constituyen la fuente de financiación. Los fondos provienen de personas comprometidas con una economía solidaria que, a su vez, adquieren los outputs de estos negocios.

\section{$2^{\circ}$ - Elaboración de un cuestionario.}

\section{Cuestionario}

- Introducción y proceso de creación

- Nos gustaría que te presentaras, quién eres, a qué te dedicas

- ¿Desde cuándo llevas con la actividad?

- ¿Es la primera empresa que has dirigido?

- ¿Con qué experiencia previa contabas?

- ¿Cuál era tu relación previa con la actividad que desarrollas ahora?

- ¿Qué motivos te llevaron principalmente por desarrollar una actividad por tu cuenta?

- ¿Cuáles han sido para ti las principales trabas o problemas que has encontrado a lo largo del camino?

- ¿Cuáles han sido tus principales aliados en el proceso?

- ¿Qué papel juegan los cursos de formación y asistencia a congresos, foros, jornadas? para mantenerse al día y establecer contactos?

- Sobre la actividad y su futuro

- ¿Cómo describirías tu modelo de negocio?, qué te diferencia del resto?

- ¿Cuáles consideras que son los principales puntos fuertes y débiles de tu negocio?

- ¿A qué amenazas o a qué oportunidades consideras que la empresa se enfrenta?

- En relación con el futuro, ¿cuáles son tus próximos proyectos?

- ¿Cuáles consideras que a tu juicio son los principales problemas a los cuales se enfrenta la mujer si quisiera desarrollar una actividad empresarial por cuenta propia?

\section{$3^{\circ}$ - Realización de las entrevistas}

- Desarrollo de entrevista semi-estructurada.

La duración de las entrevistas osciló entre los 30 minutos, de la más corta, hasta la hora y 10 minutos de la más larga. 
- Trascripción de entrevistas.

Como siguiente paso se procedió a la transcripición literal de las grabaciones en soporte audio.

\section{$4^{\text {o }}$ - Elaboración y redacción de casos y adecuación de los casos para plasmarlos en el material docente.}

\section{$5^{\circ}-\quad$ Publicación de un libro}

El producto final elaborado a partir de este estudio ha sido la elaboración de un libro llamado Creación de empresas y género: casos reales de mujeres emprendedoras. Dicho libro se encuentra en proceso de edición y es coeditado por el consejo social de la universidad de Córdoba y la editorial Tirant lo Blanc (indexada en el primer cuartil del índice SPI).

\section{LÍNEAS FUTURAS DE ACTUACIÓN}

Durante el curso 2018-2019 se comenzará a emplear el libro de casos como material docente, de manera incial en en las asignaturas de Creación de Empresas (Grado en Administración y Dirección de Empresas y Doble Grado en Derecho y Administración de Empresas), y posteriormente en las asignaturas de Creación de Empresas Turísticas (Grado en Turismo) y Creación de Empresas Culturales (Grado de Gestión Cultural). Esto posibilitará cumplir con los objetivos previstos relativos a la mejora de las capacidades y habilidades respecto del proceso emprendedor a la visibilización y sensibilización de casos emprendimiento femenino.

Asímismo, el objetivo es medir la vocación emprendora exante y expost por medio de un cuestionario, tanto al inicio como al final de la asignatura y si existen diferencias significativas entre la vocación emprendedora femenina y masculina. Concretamente, se pretende, basándonos en los trabajos Veciana y Urbano, medir cuál es el comportamiento del alumnado con respecto a su propensión y actitud hacia el autoempleo el que se tratará de contrastar la siguiente hipótesis:

H.1. La propensión a la actividad emprendedora entre hombres y mujeres se ha visto reducida tras la implantación de los actividades propuestas.

Esta hipótesis general se fragmentará a su vez en otras que identifican el efecto de las actividades sobre la autopercepción del alumnado sobre una serie de atributos personales (creatividad, dinamismo, etc.) así como el grado de valoración de una serie de profesiones (entre ellas la de empresario).

Por otra parte, se busca observar si existen diferencias entre ambos grupos de estudiantes (chicos y chicas), en relación con su formación sobre la materia, así como con su comportamiento y actitud frente a determinadas cuestiones relacionadas con el género. Además, se trataría de ver, si existe diferencia, en qué medida puede derivarse de la formación recibida.

Uno de los resultados esperados con esta experiencia es el de encontrar alumnas que se planteen al término de sus estudios llevar a la práctica alguna idea de negocio. La simple intención de transformar una idea en una empresa tangible indica el proceso transformador que poco a poco se abre paso en las intenciones emprendedoras de nuestras alumnas.

\section{CONCLUSIONES}

El material docente elaborado petende tener una doble finalidad: por un lado, de forma inmediata, pretende sensibilizar al alumnado en cuestiones de género, visibilizando el papel de la mujer en el ámbito empresarial; y por otro, a medio/largo plazo, desarrollar competencias transversales tan importantes como son el empredimiento y la autonomía personal en la toma de decisiones.

El desarrollo del proyecto ha permitido construir un material docente de calidad que enriquece y acompaña el proceso de enseñanza y aprendizaje. Todas las emprendedoras se han decantado por la opción del emprendimiento no sólo por motivos económicos, ni por las aspiraciones tradicionales de ganar dinero, sino que buscaban la realización personal a través de un proyecto propio, y como elección propia por un tipo de empleo más flexible, sociable y creativo. Todo ello permitió un acercamiento del alumnado al mundo de la emprendedora y sus distintas facetas, potenciando de este modo un mayor y mejor conocimiento del mismo.

Como última recomendación consideramos que esta metodología u otras basadas en principios parecidos deben de fomentarse dentro de la comunidad universitaria para lograr que el alumnado llegue a alcanzar las 
competencias establecidas y podamos alcanzar una mayor igualdad de oportunidades entre egresados y egresadas universitarios. Por lo tanto, estas nuevas metodologías han de complementar (cuando no sustituir) paulativamente a las tradicionales para la adquisición de competencias específicas.

\section{AGRADECIMIENTOS}

Este trabajo ha sido financiado a través de la convocatoria del "Plan de Innovación y Buenas Prácticas Docentes 2016/2017”".

Queremos ofrecer nuestro agradecimiento a cada una de las personas que se han ofrecido a participar en nuestro proyecto. Especialmente a aquellas emprendedoras que de manera altruista han decido parte de su tiempo a ser entrevistadas Asofi Asesores; Cynara Asesores; Gliquet; Gourmet Jienense; Hoy no cocino; Innovación Creativa; La otra cafetería; La voz de Marta; Las Aventuras de Tate; Pollos de la aldea y Sopinet.

Así mismo queremos agradecer la colaboración a la unidad de Empleo de Cruz Roja Española en Córdoba, así como a $\mathrm{M}^{\mathrm{a}}$ Rosario Fernández Pérez, cooperativista de Fiare, Banca Ética y socia miembro de FIARE Castilla y León, por facilitarnos algunos de los contactos con las emprendedoras entrevistadas para la construcción de casos de estudio.

\section{BIBLIOGRAFÍA}

Baughn, C. C., Cao, J. S., Le, L. T. M., Lim, V. A., y Neupert, K. E. (2006). Normative, social and cognitive predictors of entrepreneurial interest in China, Vietnam and the Philippines. Journal of developmental entrepreneurship, 11(01), 57-77.

Berg, B. y Lune, H. (2012). Qualitative research methods for the social sciences, 8th International Edition, New Jersey: Pearson.

Díaz Casero, J.C.; Hernández Mogollón, R. y Raposo, M. (2007): “Estudiantes universitarios y creación de empresas. Un análisis comparativo entre España y Portugal". En Ayala Calvo (coord.): Conocimiento, innovación y emprendedores: Camino al futuro, 1338-1352.

Hernangómez-Barahona, Juan; Martín-Cruz, Natalia y Martín-Pérez, Víctor (2006), La relevancia del objetivo en la medida de la ciencia. Un análisis para las ONGD españolas desde la teoría del comportamiento. Boletín Económico del ICE, Volumen 2884, España, Ministerio de Economía y Competitividad, pp 17-30.

Justo, R., y Díaz, C. (2012). Incidencia de los Modelos de Referencia en la Creación de Empresas: Efectos Mediadores y de Género. Economia industrial, (383), 111-124.

Langowitz, N., y Minniti, M. (2007). The entrepreneurial propensity of women. Entrepreneurship theory and practice, 31(3), 341-364.

Lim, S., y Envick, B. R. (2013). Gender and entrepreneurial orientation: a multi-country study. International Entrepreneurship and Management Journal, 9(3), 465-482.

Minniti, M. (2004). Entrepreneurial alertness and asymmetric information in a spin-glass model. Journal of Business Venturing, 19(5), 637-658.

Naciones Unidas (2015). Transforming our world: the 2030 Agenda for Sustainable Development. $\mathrm{A} / \mathrm{RES} / 70 / 1$.

Sánchez Cañizares, S. M., y Fuentes García, F. J. (2010). Gender differences in entrepreneurial attitudes. Equality, Diversity and Inclusion: An International Journal, 29(8), 766-786.

Van Dam, N. (2004). The e-Learning Fieldbook. USA: McGraw-Hill.

Veciana, J.M., Aponte, M. y Urbano, D. (2005): “University students' attitudes towards entrepreneurship: a two countries comparison”, International Entrepreneurship and Management Journal, 1 (2), 165-182.

Ventura Fernández, R., y Quero Gervilla, M. J. (2013). Factores explicativos de la intención de emprender en la mujer. Aspectos diferenciales en la población universitaria según la variable género. Cuadernos de gestión, 13(1).

Wilson, F., Kickul, J., y Marlino, D. (2007). Gender, entrepreneurial self-efficacy, and entrepreneurial career intentions: implications for entrepreneurship education. Entrepreneurship theory and practice, 31(3), 387-406.

Yin, R. K. (2003). Case study research design and methods third edition. Applied social research methods series, 5. 